\title{
An Overview of the Importance of Bacterial Elements for Plant Genetic Engineering
}

da Cunha NB', Leite ML ${ }^{1,2}$, de Loiola Costa LS ${ }^{1}$, Cunha VA ${ }^{1}$, Sena Macedo $\mathbf{M W F}^{\mathbf{1}}$ and Dias $\mathbf{S C}^{\mathbf{1}}$

${ }^{1}$ Center for Biochemical and Proteomic Analysis and Postgraduate Program in Genomic Sciences and

Biotechnology, Catholic University of Brasília, Brasília ${ }^{2}$ Department of Molecular Biology, University of Brasilia, Brasilia/ DF, Brazil

*Corresponding author: Nicolau Brito da Cunha, Center for Biochemical and Proteomic Analysis, Catholic University of Brasilia, Brasilia/ DF, Brazil. Postgraduate Program in Genomic Sciences and Biotechnology, Catholic University of Brasília, Brasília/ DF, Brazil. SGAN 916, Av. W5, Módulo C, Sala 219, Brasilia - DF, Brazil

Received: December 24, 2020; Accepted: J anuary 19, 2021; Published: J anuary 26, 2021

\begin{abstract}
Plant genetic engineering is one of the most important aspects of biotechnology applied to plant systems. The stable introduction of exogenous genetic material in plant cells is a determining step for obtaining transgenic plants. In this context, bacteria are crucial for the development of transgenic plants. Gene cloning often involves the use of bacterial plasmids and DNAmodifying enzymes synthesized in genetically modified bacteria. In addition, among the several methods of introducing genes into plants, the method that uses Agrobacterium tumefaciens continues to be used to obtain genetically modified plants for the agricultural, pharmaceutical and materials industry sectors. This minireview aims to present the basic aspects of bacterial elements related gene manipulation for obtaining transgenic plants.
\end{abstract}

Keywords: Biotechnology; Plant Genetic Engineering; Gene

\section{Introduction}

As molecular units that contain the genetic information expressed in RNA and proteins, genes were the target of new uses and biological approaches in the early 1980s, from the discovery that certain organisms, notably bacteria, naturally have the ability to transfer genes for plants, changing the phenotype of the latter. The understanding of the main molecular mechanisms that govern the genetic transformation of plants opened precedents for the in vitro transfer of genes between different organisms, commonly called transgenics. In this context, we can define transgenia and transgene respectively as i) the incorporation of exogenous and inheritable DNA by living organisms through in vitro methods ans ii) genes originating from different organisms (they may even belong to species that do not have sexual compatibility), usually obtained through recombinant DNA technology and introduced naturally or in the laboratory into the genome of recipient species [1].

In this way, transgenic organisms such as bacteria, yeast cells, filamentous fungi, plants and animals, are those that contain one or more genes inserted in their genomes by means other than the sexual route. The cells of these organisms are able to coordinate transcription of transgenes and also the translation of mature mRNAs into proteins correctly folded, assembled and modified after translation. In this case, the new proteins produced - called recombinant proteins are capable of desirably altering the phenotype of gene receptor organisms in order to achieve a series of practical objectives [1].

Recombinant protein is can be defined as a polypeptide encoded by a transgene and normally synthesized in organisms other than those of its origin. Some recombinant proteins can be encoded by additional copies of transgenes from the host plant itself, introduced in vitro into its genome [2].

When the development of transgenic plants, as well as other genetically modified organisms, meets objectives related to food production or improved obtaining drugs, for example, we can say that they are essentially biotechnological products. In this context, Biotechnology can be defined as the various forms of technology that exploit biological sources - usually microorganisms and/or their products and components. The term is also commonly used to define the forms of technology that depend on the use of molecular biology/ genetic engineering techniques for the construction of new organisms and/or products for industrial, medical and other applications [3].

The molecular strategies that advocate the recombinant protein biosynthesis, mediated by the enzymatic machinery of higher plants, are the applied examples that arrived on the market and those most frequently used by research groups involving the genetic transformation of plants. A significant portion of the research involving transgenic plants is restricted to fundamental studies of experimental validation, prior to obtaining a product intended for the market. The herbaceous plant Arabidopsis thaliana (L.) Heynh. a crucifer evolving closely to the mustard plant [Brassica integrifolia $(\mathrm{H}$. West) Rupr.], is inserted in the latter case, since it received the status of model plant for experimenting with plant transgenics in a manner analogous to the position reached by flies of the genus Drosophila sp. for the study of population genetics [3].

Whether as a result of the expression of one or more recombinant proteins or by the suppression/blocking of the expression of proteins and metabolic intermediates of the plant itself or of infesting pathogens, the objectives that govern obtaining transgenic plants for the market are mainly focused on obtaining food, medicines and industrial products and reagents [4]

The ability of higher plants to express genes from other organisms (not necessarily close to the evolutionary point of view) and to produce recombinant proteins in satisfactory quantities and in an economically viable manner are the main factors that characterize them as efficient systems for the production of numerous proteins with the most varied applications. The uses of recombinant proteins 
of plant origin are divided into two major groups: endogenous and exogenous. Endogenous use is that which is restricted to the organism of origin of the recombinant protein. Exogenous use is one whose purpose concerns other organisms than that of origin of a recombinant protein [5].

Endogenous uses, that is, in the transgenic plant itself, is usually related to agronomic and recurrent aspects of human food, such as obtaining resistance to forms of biotic stress, such as the attack of insects and pathogens that cause diseases that compromise crops and the storage of agricultural products; obtaining tolerance to different forms of abiotic stress - mainly to the toxic action of herbicides - and the so-called "functional" foods, which have higher yields of production of certain oils, vitamins, amino acids and carbohydrates and which have greater nutritional value and desirable characteristics to the human and animal diet, such as the decrease in antinutritional/ allergenic and undesirable factors for the food industry [4-6].

Transgenic plants also function as vehicles for the production of proteins and other biomolecules to be extracted from plant material and purified for their exogenous application, that is, outside the plant in which they were biosynthesized. The destinations for using these true protein bio-factories are numerous, ranging from the pharmaceutical industry to that of cleaning and hygiene materials, including the development of recombinant enzymes for strictly industrial application and also to obtain new materials (biopolymers) with desirable characteristics for the clothing, surgical and potential employment sectors in the security, naval, air and military sectors [6].

Obtaining transgenic plants is based on three broad sets of cellular and molecular techniques: genetic engineering, techniques for transferring genes between different organisms and in vitro culture of plant tissues. Genetic engineering is a comprehensive set of concepts, techniques and molecular applications carried out from the first half of the 1970s and developed by the progressive correlation of important information for understanding the structure and function of nucleic acids, obtained over thirty years before, and by the discovery of enzymes capable of modifying the molecular structure of nucleic acids, which allowed their manipulation in vitro. Genetic engineering allows the controlled modification of nucleic acid molecules in the laboratory, to generate new combinations of genes or sequences (such as fusion proteins) [7].

Genetic engineering techniques allow the assembly, in the laboratory, of hybrid DNA molecules, containing functional segments of deoxyribonucleic acids from more than one organism structured in an organized way, as in a "molecular mosaic", in which multiple stretches of DNA are assembled in logically and that allows each segment to perform its function correctly [2-4].

The tools used to manipulate the DNA of different organisms are enzymes capable of structurally modifying these molecules and integrating recombinant fragments (containing different combinations of DNA - hence the name recombinant DNA technology) in circular bacterial DNA molecules called plasmids [1].

Plasmid is a bacterial extrachromosomal genetic element, often transmissible from cell to cell, which can be propagated both in the cytoplasm and as an integral part of the bacterial chromosome. These circular DNA molecules are not essential for the bacterium's survival, but contain genes normally associated with important phenotypic traits, such as antibiotic resistance. Its application is recurrent in genetic engineering in the cloning and expression of transgenes [1-4].

The DNA-modifying enzymes mentioned earlier catalyze processes of alteration at the structural level in the molecule, such as restriction and cleavage at specific sequential sites of nitrogenous nucleotide bases and the binding of compatible DNA ends $[4,8]$.

The first of these enzymes was purified from cells of the bacterium Escherichia coli and characterized in 1967 by Martin Gellert, of the National Institutes of Health (NIH) and called DNA ligase. Gellert found that this enzyme was able to catalyze the establishment of phosphodiester bonds between different fragments of lambda phage $(\lambda)$ DNA, linking different parts of the virus's DNA molecule $[5,8]$.

Just a year later, biochemists Stuart Linn and Werner Arber, from the University of Geneva (Switzerland), discovered the catalytic action of DNA restriction enzymes, culminating in the purification and characterization of the first site-specific restriction endonuclease - endonuclease R, later called Hind II $[4,8]$.

The use of both classes of enzymes allows for the specific fragmentation of DNA molecules carrying the restriction sites recognized by the endonuclease, in addition to the binding of the ends of different molecules by reconstituting the covalent fodder-type bonds between the phosphate groups and the deoxyribose molecules from both ends, a mechanism effected by the catalytic action of the enzyme DNA ligase $[3,8]$.

The same procedure can be used to insert fragments containing transgenes - including those that confer resistance to antibiotics - in the bacterial plasmids themselves previously isolated in the laboratory [8]. Plasmids containing transgenes are then re-introduced into bacteria that accept them as elements of their own genome. Once the transgenic bacterial cells (carrying the modified plasmid) can replicate under suitable conditions, in a selective culture medium containing the antibiotic against which they are resistant, the plasmids carrying the transgenes also undergo a replication process, resulting in the obtaining of new copies of plasmid DNA. Each replicated bacterial clone contains multiple copies of the plasmid and, similarly, of the transgene itself. This mechanism was called gene cloning $[5,8]$.

Once multiplied on a large scale, the various copies of the plasmids containing the transgenes are separated from the bacterial cells and purified in vitro, in order to be free of contaminating molecules, for their later introduction into the nucleus or lumen of plant organelles containing their own genomes, such as mitochondria and chloroplasts [8].

Bacterial plasmids modified by genetic engineering and used in experiments of genetic transformation of plants are also called plasmid vectors of gene expression, since they contain the coding sequences of recombinant proteins properly flanked by different regulatory sequences, as promoters; signal peptides and terminators which, once arranged sequentially and in the same reading phase, constitute the expression cassette to be stably integrated into the plant genome $[3,4]$.

The expression cassette consists of one or more genes and their flanking regulatory sequences. Generally, its constituents are the 
promoter of the gene, an open reading phase (the coding sequence) and the DNA sequence corresponding to the 3 'untranslated region of the mRNA, which contains the polyadenylation site in eukaryote. The promoter of a gene is a region of DNA directly involved in the initiation of gene transcription, located upstream, on the same strand and generally close to the coding sequence it regulates. Present at the promoter are the site of initiation of RNA synthesis and the sites and responsive binding elements of RNA polymerase dependent on DNA and transcription factors, as well as regulatory elements such as activators and repressors of transcription [8].

The signal peptide sequence is a small sequence encoding a short peptide chain, directly involved with the post-translational targeting and transport of a protein to organelles or to the secretory pathway of proteins in transit in eukaryotic cells. They can be associated with both the $\mathrm{N}$ and the $\mathrm{C}$-terminus of the target protein. Some signal peptides undergo specific enzymatic recognition and cleavage after protein transport. A gene's coding sequence is the portion of nucleic acid that is transcribed into RNA, which may or may not be translated into a protein. The transcription terminator is the region that marks the end of the gene and contains the transcription stop signal $[1,2]$.

DNA cloning techniques were first developed and executed in 1972 by Paul Berg at Stanford University, California, and resulted in the obtaining of a hybrid DNA molecule, containing a stretch of DNA sequence from a Lambda phage $(\lambda)$ inserted into the genome of the SV40 tumor-causing virus, which could be inserted into mammalian cells $[3,5]$.

Just a year after Berg's achievement, Stanley Cohen, at the same university, was able to develop a method of inserting exogenous bacterial plasmids into recipient $E$. coli cells and, together with Herbert Boyer of the University of California, reported the first in vitro construction of a bacterial plasmid containing genes from two other plasmids previously digested with the restriction enzyme EcoRI Once inserted in E. coli cells, the hybrid plasmid of Boyer and Cohen was efficient in giving the transgenic bacteria simultaneous tolerance to antibiotics tetracycline and kanamycin [8].

The second premise for obtaining transgenic plants is the transfer of genes of interest from other organisms to plant cells potentially precursor to an organ or an embryo. The genetic transformation of plants can be understood as the controlled introduction of genes from different animal, plant or microorganism species, into a recipient plant genome, regardless of fertilization [1-5].

Despite the development of different techniques of plant genetic transformation, the introduction of genes of interest in higher plants has been carried out conventionally by two processes: through the infection of target tissues by transgenic cells of the bacterium $A$. tumefaciens - method preferably used for the transformation genetics of most dicotyledonous plants - or by bombarding explants with microparticles containing DNA carrying the genes of interest that are superficially adsorbed (a method known as biobalistics) [8].

This last method, in addition to demonstrating less genotypic dependence, is the most used for the genetic transformation of legumes, such as soybeans (Glycine max) and alfalfa (Medicago sativa L.) and cereals, such as rice (Oriza sativa), wheat (Triticum aestivum) and corn (Zea mays) [9].

\section{The method that uses Agrobacterium tumefaciens}

Genetic transformation by agrobacteria is based on the capacity that the species A. tumefaciens, a bacterium typically from the soil, aerobic, in the form of a bacillus, Gram-negative, non-spore-forming and etiological agent of plant disease known as crown gall, has in transferring part of the DNA of its plasmid $\mathrm{Ti}$ (from Tumor inducing) to the genome of cells of host plants [10].

In order to take advantage of the potential for transferring genes from A. tumefaciens to higher plants, a methodology for cultivating "unarmed" strains of the bacterium was developed, which had, by double recombination, deleted the oncogenes from a region of 150 to $250 \mathrm{~kb}$ of the plasmid Ti, called T-DNA. The right and left ends of the T-DNA, essential for the transfer, are kept intact and between them the sequence containing the gene of interest to be introduced into the plant is cloned $[8,9]$.

Another region of the bacterial Ti plasmid, called the virulence region (vir regulon region with approximately 25 genes), encodes proteins that promote the transfer of the T-DNA region containing the genes of interest to the plant cell genome. Thus, it is necessary to carry out a co-culture based on the cultivation of the explant to be transformed together with an unarmed strain of A. tumefaciens carrying the plasmid containing the genes of interest to be transferred to the plant $[8,9]$.

The agroinfection method was adopted to obtain the first transgenic plant, developed by researchers from the group of Luis Herrera-Estrella and Patricia Zambryski at the University of Gent (Belgium) in 1984 - a tobacco plant (Nicotiana. tabacum) containing a gene bacterial resistance to kanamycin antibiotic [8].

\section{The method of accelerating microparticles covered with DNA}

The biobalistic process recommends the acceleration of microprojectiles (from 0.2 to $4.0 \mu \mathrm{m}$ in diameter) covered with sequences of nucleic acids, at a final speed of $1,500 \mathrm{~km} / \mathrm{h}$, on the tissue segments or plant organs to be transformed. The microprojectiles, of gold or tungsten, penetrate non-lethally the cell wall and the cytoplasmic membrane of the pumped cells, lodging themselves randomly in the cell organelles, among which the nucleus, mitochondria and chloroplasts, when then the DNA is dissociated from microprojectiles by the action of intra-organellar liquid content and integration by homologous recombination of the sequence containing the genes of interest to the plant genome [10].

A wide variety of microprojectile acceleration systems have already been developed, most of which share the idea of generating a shock wave carrying enough energy to displace a carrier membrane containing the microprojectiles coated with DNA [10].

The shock wave can be generated by a chemical explosion, by a discharge of helium at high pressure, by the vaporization of a drop of water by electrical discharge with high voltage and low capacitance or vice versa, by a discharge of compressed air or, when the use of a carrier membrane is not required, using a low pressure helium gas discharge. The system that uses helium gas under high pressure is the one that is more efficient in obtaining high transformation frequencies, in addition to being widely used for the genetic transformation of a very large variety of plant species [10-15]. 


\section{In vitro culture of plant tissues}

In most cases, the choice of transgenic plants for the production of any class of protein focuses preferably on the stable genetic transformation and regeneration of complete plants from explants. Explants are segments of tissue or plant organs used to initiate an in vitro culture $[1,16]$.

As transgenic plants are regenerated in vitro from previously transformed germ or totipotent cells, all cells of the resulting individuals are carriers of the exogenous nucleotide sequence (transgene), being able to transmit copies to their offspring by the same principles that govern heredity in non- transgenic plants [10]

In vitro morphogenesis is based on the totipotence of plant cells and the ability to grow these cells in the laboratory, through the use of nutrient culture media and growth regulators appropriate to modulate plant regeneration. Two models of in vitro morphogenesis and its variations are used for the regeneration of plants for their genetic transformation: organogenesis and somatic embryogenesis [10].

Organogenesis stimulates the formation of aerial parts or roots in callus culture (mass of cells of continuous proliferation and more or less disorderly) or other explants, from the neoformation of vegetative or floral stem buds that become stem axes or roots. In this method, the regenerated organs have a multicellular and subepidermal origin and regeneration occurs from meristematic cell groups of the original tissue [10].

The cultivation of embryos with their apical meristematic region preserved or simply from the apical or axillary meristem systems, in the presence of cytokinins in culture medium, is an efficient inducer of aerial part organogenesis, capable of regenerating transgenic plants without the need for passage of the tissue by intermediate stages of de-differentiation, such as callus, for example [10].

Somatic embryogenesis recommends the development of embryos from somatic cells, as a result of an external stimulus (contact with growth regulators, such as 2,4-D - 2,4 dichlorophenoxyacetic acid). This tissue culture method leads to adventitious multi-embryonic formation of explants, and the originated embryos have their own vascular axes, single-cell or few cell histological origin and superficial location [10].

The regeneration process of plants undergoing genetic transformation via biobalistic reduces the contact period between the explant and hormonal phyto-regulators, since the tissue is not necessarily submitted to the callus phase, a characteristic that makes the process of obtaining transformants faster and under more intensified control conditions [10].

Another important advantage of the biobalistic system is the reduction of in vitro culture time, providing molecular and biochemical tests for the identification of transforming events and proteins with greater precocity. Both forms of plant regeneration in vitro allow, from the initial explants, the continuous obtaining of tissues and experimental material through cyclic cultures, which once associated with other tissue culture techniques have fundamental applications and importance in several areas as in the genetic improvement of plants, in phytopathology and in the genetic transformation of plants [17].
As a result of the integrated action between the three sets of the main techniques that constitute the process of genetic transformation of plants, it is possible to build plasmid vectors containing genes of interest, perform their introduction in plant cells and explants, verify the integration of exogenous sequences to the genome of plant species in question and express them stably in successive generations, in order to obtain transgenic plants and recombinant proteins in significant quantities [17].

\section{Conclusion}

Transgenic plants can function efficiently as vehicles for the expression of recombinant proteins and, in many cases, promote high levels of accumulation of recombinant production, through different molecular strategies. The action of DNA-modifying enzymes, often biosynthesized by genetically modified bacteria, is essential for the manipulation of DNA fragments containing the genes of interest and other elements of the expression cassette.

A. tumefaciens, like biobalistics, are the main methods of genetic transformation of plants. An interesting aspect of agroinfection is the greater control of the number of copies inserted in the genome of the host plant, thanks to the relationship of biological compatibility between bacteria/plant and the type of integration directed by the end of the expression cassette cloned in the Ti plasmid.

Regardless of the genetic transformation method and the type of regulatory sequences present in the plasmid expression vector, the levels of gene expression are directly related to the choice of the compartment for the accumulation of recombinant proteins, posttranslational protein stability and adaptation at the genetic level of sequences capable of minimizing proteolytic degradation in the various stages of modification after translation.

In principle, it takes a long time to optimize the expression of a recombinant protein in any biological production system, especially when different subcellular compartments are available, such as those observed in plant cells.

Once the appropriate combination involving the type of plant receiving the transgene has been reached, the different elements of regulation of gene expression in the expression cassette, the molecular strategy of subcellular protein addressing and the protocols for genetic transformation and tissue culture, the capacity The plant's functioning as a correct, safe and economical agent of biosynthesis of a given recombinant protein is put in check over several stages until effectively exercising direct or indirect influences on the consumer's quality of life.

\section{Acknowledgment}

This work was supported by the Coordination for the Improvement of Higher Education Personnel (CAPES), the National Research and Development Council (CNPq) and the Research Support Foundation of the Federal District (FAPDF).

\section{References}

1. Aragao FJL, Barros LMG, de Sousa MV, de Sa MFG, Almeida ERP, Gande $\mathrm{ES}$, et al. Expression of a methionine-rich storage albumin from the Brazi nut (Bertholletia excelsa HBK, Lecythidaceae) in transgenic bean plants (Phaseolus vulgaris L, Fabaceae). Genet Mol Biol. 1999; 22: 445-449.

2. Aragao FJL, Barros LMG, Brasileiro ACM, Ribeiro SG, Smith FD, Sanford 
JC, et al. Inheritance of foreign genes in transgenic bean (Phaseolus vulgaris L) co-transformed via particle bombardment. TAG Theor Appl Genet. 1996; 93:142-150.

3. Jackson DA, Symons RP, Berg P. Biochemical Method for Inserting New Genetic Information into SV40 DNA: Circular SV40 DNA Molecules Containing Lambda Phage Genes and the Galactose Operon of Escherichia coli. Proc Nat Sci USA. 1972; 69: 2904-2909.

4. Goff SP, Berg P. Construction of Hybrid Viruses Containing SV40 and Lambda Phage DNA Segments and Their Propagation in Cultured Monkey Cells. Cell. 1976; 9: 695-705.

5. Mulligan RC, Howard BH, Berg P. Synthesis of Rabbit Beta-Globin in Cultured Monkey Kidney Cells Following Infection with a SV40 beta-Globin Recombinant Genome. Nature. 1979; 277: 108-114.

6. Berg P, Mertz JE. Personal Reflections on the Origins and Emergence of Recombinant DNA Technology. Genetics. 2010; 184: 9-17.

7. Capell $\mathrm{T}$, Christou $\mathrm{P}$. Progress in plant metabolic engineering. Curr Opin Biotechnol. 2004; 15: 148-154.

8. Nicholl DST. An Introduction to Genetic Engineering. $2^{\text {nd }}$ ed. UK: Cambridge University Press. 2002

9. Kusnadi AR, Zivko L, Nikolov JAH. Production of recombinant proteins in transgenic plants: Practical considerations. Biotechnol Bioeng. 1997; 56 473-484.
10. Rech EL, Vianna GR, Aragao FJL. High-efficiency transformation by biolistics of soybean, common bean and cotton transgenic plants. Nat Protoc. 2008; 3: $410-418$

11. Fischer R, Stoger E, Schillberg S, Christou P, Twyman RM. Plant-based production of biopharmaceuticals. Curr Opin Plant Biol. 2004; 7: 152-158.

12. Hood EE, Jilka JM. Plant-based production of xenogenic proteins. Curr Opin Biotechnol. 1999; 10: 382-386.

13. Daniell H. GM crops: public perception and scientific solutions. Trends Plant Sci. 1999; 4: 467-469.

14. Chapman MA, Burke JM. Letting the gene out of the bottle: the population genetics of genetically modified crops. New Phytol. 2006; 170: 429-443.

15. Daniell $\mathrm{H}$. Molecular strategies for gene containment in transgenic crops. Nat Biotechnol. 2002; 20: 581-586.

16. Stoger E, Ma JKC, Fischer R, et al. Sowing the seeds of success: pharmaceutical proteins from plants. Curr Opin Biotechnol. 2005; 16: 167173.

17. Twyman RM, Stoger E, Schillberg S, Christou P, Fischer R. Molecular farming in plants: host systems and expression technology. Trends Biotechnol. 2003; 21: $570-578$ 